\title{
Controle de infecção: um requisito essencial na prática da acupuntura - revisão de literatura ${ }^{1}$
}

\section{Infection control: an essential requirement in acupuncture practice - review of the literature}

\section{Control de la infección: un requisito esencial de la práctica de la acupuntura - revisión de la literatura}

\author{
Flaviana Regina Pimental', Lara Stefania Netto de Oliveira Leão", Fabiana Cristina Pimenta"
}

\begin{abstract}
RESUMO
A acupuntura é uma técnica milenar de origem chinesa, tem seu uso recomendado pela Organização Mundial da Saúde (OMS) e foi incorporada no Sistema Único de Saúde (SUS). Devido sua grande expansão, o objetivo desse estudo foi verificar, por meio de uma revisão bibliográfica, a segurança e o controle de infecção na prática da acupuntura, identificando possíveis riscos e efeitos adversos. A busca do material foi realizada em livros, periódicos online (PubMed, MEDLINE, LILACS e SciELO) referente aos anos de 1984 a 2007 e manuais sobre acupuntura e controle de infecção. Vários estudos comprovaram que a acupuntura é segura e eficaz, sendo que grande parte de seus efeitos adversos podem ser evitados por profissionais devidamente capacitados e treinados. Um dos principais requisitos para a realização da acupuntura é o controle de infecção, pois é uma técnica invasiva que tem como principal ferramenta o uso de agulhas, que penetram na pele, tecido subcutâneo e músculos, podendo provocar a transmissão de microrganismos. Concluiu-se que desde que todas as normas de biossegurança sejam seguidas, os riscos na prática da acupuntura são mínimos e sempre se preconiza o uso de agulhas descartáveis.
\end{abstract}

Palavras chave: Infecção; Exposição a agentes biológicos; Terapia por acupuntura, Acupuntura.

\section{ABSTRACT}

The acupuncture is a millenary technique from China, it is recommended by World Health Organization (OMS) and was incorporated in the Sistema Único de Saúde (SUS). Due this large expansion, the objective of this paper was to review the literature about security and the infection control in the acupuncture practice, and recognize possible risks and adverse effects. The data was collected in books, magazines (PubMed, MEDLINE, LILACS e
}

SciELO) in the period of 1984 to 2007 and manuals of acupuncture and infection control. Many reports showed that acupuncture is security and efficient, and the most of adverse effects could be avoid for professionals welltrained and capable. One of the major requisites during acupuncture is the infection control, because this is an invasive technique and the needles are the principal instrument, which penetrated in skin, subcutaneous tissues and muscular, providing microorganisms transmission. We concluded that if the biosecurity standards are followed, the risks in this practice will be low and disposable needles should be always used.

Key words: Infection; Exposure to biological agents; Acupuncture therapy; Acupuncture.

\section{RESUMEN}

La acupuntura es una técnica milenaria originaria de la China y su uso ha sido recomendado por la por la Organización Mundial de la Salud (OMS) y ha sido incorporado al Sistema Único de Salud (SUS). Debido a su gran expansión, el objetivo de esta investigación fue verificar por medio de una revisión bibliográfica cuales son las normas de bioseguridad y los riesgos de la acupuntura. La revisión fue realizada en libros y revistas especializadas (PubMed, MEDLINE, LILACS e SCiELO) - 1984 la 2007 y manuais de la acupuntura y el control de la infección. Varios

\footnotetext{
${ }^{1}$ Artigo extraído de dissertação de mestrado do Programa de Pós-Graduação em Ciências da Saúde da Faculdade de Medicina da Universidade Federal de Goiás (UFG).

Acupunturista, Docente do Instituto Brasileiro de Acupuntura de Ribeirão Preto - SP - IBRAMRP, mestranda no Programa de Pós-Graduação em Ciências da Saúde da Faculdade de Medicina da UFG. E-mail: prof.flaviana@ibramrp.com.br.

"Professora do Departamento de Microbiologia, Imunologia Parasitologia e Patologia do Instituto de Patologia Tropical e Saúde Pública da UFG.
} 
Pimenta FR, Leão LSNO, Pimenta FC. Controle de infecção: um requisito essencial na prática da acupuntura - revisão de

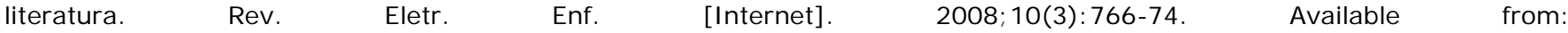
http://www.fen.ufg. br/revista/v10/n3/v10n3a22.htm.

estudios comprobaron que la acupuntura es segura y eficaz y la mayoría de sus efectos adversos pueden ser evitados por profesionales debidamente capacitados y entrenados. Uno de los principales requisitos básicos para la realización de la acupuntura es el control de la infección, pues es una técnica invasiva que tiene como principal herramienta el uso de las agujas, que penetran en la piel, tejido subcutáneo y músculos pudiendo provocar la

\section{NTRODUÇÃO}

Apesar de ser uma técnica milenar de origem chinesa, a acupuntura é um símbolo de modernidade nas civilizações ocidentais, pois sua expansão e aceitação datam de poucos anos. Possui inúmeras aplicações, sendo possível seu uso independente da faixa etária e do gênero. A acupuntura vem resgatar aquilo que muitos procuram, o tratamento integral, considerando o homem como ser bio-psicosócio-espiritual. Para a utilização dessas técnicas, há necessidade de profissionais habilitados, visando praticar a arte de curar e não simplesmente a arte de espetar.

A acupuntura é um tratamento de baixo custo com inúmeros resultados benéficos. Foi introduzida no Brasil há cerca de 100 anos, pelos imigrantes japoneses que vieram trabalhar na agricultura. Entre eles haviam alguns praticantes da Medicina Tradicional Oriental, os quais implantaram essa técnica que, então, se difundiu(1).

Os efeitos colaterais devido ao uso da acupuntura são reduzidos. Apesar de ser uma técnica eficaz e segura, recomenda-se o uso de agulhas esterilizadas e descartáveis para evitar o risco de transmissão de microrganismos ${ }^{(2-4)}$.

A Organização Mundial da Saúde (OMS) recomenda o uso da acupuntura. Várias estudos mostram sua eficácia e segurança, bem como métodos de pesquisa e avaliação dos resultados terapêuticos das medicinas complementares e tradicionais $^{(5)}$.

No Brasil, a acupuntura foi introduzida com maior ênfase há cerca de 40 anos. Em 1988, por meio da Resolução № 5/88, da Comissão Interministerial de Planejamento e Coordenação (CIPLAN), teve as suas normas fixadas para 0 atendimento nos serviços públicos de saúde. Em 1999, o Ministério da transmisión de microorganismos. Se concluye que cuando se siguen todas las normas de bioseguridad, los riesgos de su práctica son mínimos y se aconseja el uso de agujas desechables.

Palabras clave: Infección; Exposición a agentes biológicos; Terapia por acupuntura; Acupuntura.

Saúde (MS) inseriu na tabela Sistema de Informações Ambulatoriais (SIA/SUS) a consulta médica em acupuntura (código 0701234), acompanhando, posteriormente, a evolução das consultas por região e em todo país ${ }^{(5)}$.

O ministro de Estado da Saúde, interino, no uso da atribuição que lhe confere o art. 87 e considerando que o MS entende que as Práticas Integrativas e Complementares compreendem o universo de abordagens denominado pela OMS de Medicina Tradicional e Complementar/ Alternativa - MT/MCA; que a acupuntura é uma tecnologia de intervenção em saúde, inserida na Medicina Tradicional Chinesa (MTC), sistema médico complexo, que aborda de modo integral e dinâmico o processo saúde-doença no ser humano; considerando que a melhoria dos serviços, o aumento da resolutividade e o incremento de diferentes abordagens configuram, assim, prioridade do MS, tornando disponíveis opções preventivas e terapêuticas aos usuários do SUS, aprovou a Política Nacional de Práticas Integrativas e Complementares (PNPIC) no Sistema Único de Saúde, portaria número 971 , de 03 de maio de $2006^{(6)}$ :

Art. 10 Aprovar, na forma do Anexo a esta Portaria, a Política Nacional de Práticas Integrativas e Complementares (PNPIC) no Sistema Único de Saúde.

Art. 2o Definir que os órgãos e entidades do Ministério da Saúde, cujas ações se relacionem com o tema da Política ora aprovadas devam promover a elaboração ou a readequação de seus planos, programas, projetos e atividades, na conformidade das diretrizes e responsabilidades nela estabelecidas. 
Pimenta FR, Leão LSNO, Pimenta FC. Controle de infecção: um requisito essencial na prática da acupuntura - revisão de $\begin{array}{llllll}\text { literatura. } & \text { Rev. Eletr. } \quad \text { Enf. 2008; 10(3):766-74. Available }\end{array}$ http://www.fen.ufg. br/revista/v10/n3/v10n3a22.htm.

Art. 3o Esta Portaria entra em vigor na data de sua publicação.

Com o aumento da prática da acupuntura no país e a busca da população por formas de cuidar da saúde menos invasivas e mais naturais, a preocupação com os aspectos legais dessa técnica no Brasil teve início em 1984 com a criação do Projeto de Lei 3838 da Câmara dos Deputados Federais. A partir de então, os Conselhos Federais preocupados com tal prática pelos seus pares, iniciaram regulamentações próprias. O COFFITO (Fisioterapia) em 1985, o CFBM (Biomedicina) em 1986, o COFEN (Enfermagem) e o CFM (Medicina) em 1995, CFF (Farmácia) em 2000, CFFO (Fonoaudiologia) em 2001 e CFP (Psicologia) em $2002^{(1)}$.

A Medicina Tradicional Chinesa (MTC) constitui um amplo campo de conhecimento, de origem e de concepção filosófica que abrange vários setores ligados à saúde e à doença e principalmente as formas de prevenção, na qual reside toda a essência da filosofia da medicina chinesa ${ }^{(7)}$.

Segundo o Ministério da Saúde ${ }^{(5)}$ a MTC é como um sistema médico integral, que incorpora meios próprios de diagnóstico (anamnese, palpação do pulso, observação da face e língua) e várias modalidades de tratamento (acupuntura, plantas medicinais, dietoterapia, práticas corporais e mentais), baseada em uma concepção holística sobre a natureza do ser humano e as suas relações com o mundo que o cerca e do qual faz parte. Dentre as várias técnicas da MTC, destaca-se a acupuntura, considerada como uma das técnicas complementares mais usadas, podendo ser aplicada paralelamente aos tratamentos convencionais alopáticos ou até mesmo de forma isolada. Considerada uma técnica milenar, a acupuntura surgiu na China em plena Idade da Pedra, há aproximadamente 4500 $\operatorname{anos}^{(7)}$.

Os procedimentos de acupuntura são atos predominantemente invasivos, que visam provocar estímulos em zonas neurorreativas de localização anatômica definida, com a finalidade de obter resposta de promoção de analgesia, restauração de funções orgânicas e modulação imune. Esses estímulos são provocados, em geral, por agulhas de acupuntura sistêmicas: instrumento filiforme perfurante, de ponta divulsionante não cortante, de dimensões e calibres variados, destinado à penetração em pele, tecido subcutâneo e tecido muscular, visando atingir a zona neurorreativa, que são chamados de pontos de acupuntura ${ }^{(5)}$.

Idealizada dentro do contexto de terapias naturalista da filosofia do Tao, na acupuntura a concepção dos canais de energia e dos pontos de acupuntura, o diagnóstico energético e o tratamento baseiam-se nos preceitos do Yin e do Yang, dos cinco movimentos (madeira, fogo, terra, metal, água), da energia (Qi) e do sangue (Xue). Essa energia, denominada de Qi, é a forma imaterial que promove o dinamismo, a atividade de todas as estruturas do organismo do ser vivo. A acupuntura visa restabelecer a circulação correta dessa energia nos canais de energia (chamados de meridianos) e nos órgãos e vísceras (Zang Fu), levando o corpo a uma harmonia de energia e matéria, sendo esse sistema muito complexo e eficaz ${ }^{(3,7)}$.

Os pontos de acupuntura são definidos pelo Ministério da Saúde ${ }^{(5)}$ como: "Regiões específicas do corpo humano e de animais, de localização bem definida através de referenciais anatômicos, situadas abaixo do tecido celular subcutâneo, na proximidade de fáscia, tecidos musculares e muitas vezes periósteo, com propriedades neurorreativas que desencadeiam estímulo terapêutico; envolvem diversas estruturas subjacentes - nervos, vasos sangüíneos, músculos, tendões, fáscias, ligamentos, ossos, cápsulas articulares - a profundidades variáveis (de poucos milímetros até mais de dez centímetros); apresentam projeção sobre a superfície cutânea, também de localização bem definida, de dimensão diminuta (cerca de $3 \mathrm{~mm}^{2}$ ), projeção esta que serve de ponto de aplicação para penetração da agulha de acupuntura em direção àquelas regiões neurorreativas. São usados também para fins diagnósticos, sinalizando a existência de diversas condições patológicas quando se apresentem com alterações estruturais ou em sua sensibilidade".

Devido a grande expansão da acupuntura e do aumento de sua popularidade, surge a 
Pimenta FR, Leão LSNO, Pimenta FC. Controle de infecção: um requisito essencial na prática da acupuntura - revisão de

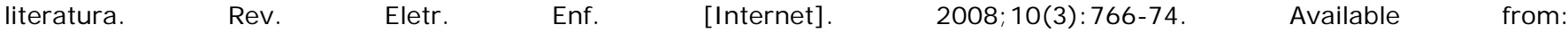
http://www. fen.ufg. br/revista/v10/n3/v10n3a22.htm.

necessidade de certificar se esta é uma terapia segura e eficaz. As agulhas sistêmicas, um perfuro-cortante, representam a sua principal ferramenta para estímulo dos pontos de acupuntura. Dessa forma, por serem procedimentos invasivos, é essencial que se preconize o controle de infecção, seguindo todas as normas estabelecidas de biossegurança.

Com base no exposto, o presente trabalho foi proposto com o objetivo de verificar por meio de uma revisão de literatura, com periódicos entre 1984 a 2007, a segurança e o controle de infecção na prática da acupuntura, identificando possíveis riscos e efeitos adversos durante os procedimentos.

\section{METODOLOGI A}

Trata-se de um estudo de revisão narrativa de literatura, desenvolvido com base em materiais já elaborados, constituído de periódicos em língua inglesa e portuguesa, manuais, livros e publicações do Ministério da Saúde (MS). As fontes de buscas usadas na pesquisa dos artigos foram em bases de dados on-line da PubMed (Biblioteca Nacional de Medicina), MEDLINE (Bibliografia Médica), LILACS (Literatura Latino-Americana e do Caribe em Ciências da Saúde) e SciELO (Scientific Eletronic Library on-line), referente aos anos de 1984 a 2007.

Foram utilizadas as seguintes palavras chaves, em português e inglês, para a busca de artigos nas bases de dados: normas da acupuntura, controle de infecção em acupuntura e efeitos adversos da acupuntura. Da busca realizada, foi feita a leitura e tradução do material e deu-se início à fase de análise dos mesmos. Alguns passos para essa análise foram seguidos, como a seleção do material considerando como critério de inclusão que o texto tratasse de um dos aspectos relacionados: descrição de normas para o controle de infecção; efeitos adversos relacionados à prática da acupuntura; biossegurança em acupuntura; formação dos profissionais que atuam com acupuntura e normas que regulamentam essa profissão.

As anotações sobre o texto foram realizadas somente após a leitura crítica, com transcrição exata dos dados úteis em relação ao tema abordado e citação correta das fontes bibliográficas. A análise e os resultados de cada artigo foram apresentados de forma descritiva e agrupados de acordo com o assunto.

Encontrou-se 74 textos referentes à temática, sendo que 54 foram excluídos, pois não atendiam aos critérios de inclusão. Foram selecionadas vinte referências, segundo os critérios acima mencionados, sendo um manual de biossegurança, seis periódicos sobre os efeitos adversos dessa terapia, um periódico sobre a eficiência e prática da acupuntura, duas publicações do Ministério da Saúde, quatro livros sobre acupuntura e controle de infecção, um periódico sobre a regulamentação da prática da acupuntura, dois periódicos sobre a segurança da acupuntura, dois periódicos e um guia do CDC (Center for Disease Control and Prevention) relacionados ao controle de infecção. As informações extraídas foram: a segurança e o controle de infecção na prática da acupuntura, identificando possíveis riscos e efeitos adversos.

Dentre os 20 textos selecionados, $11 \mathrm{se}$ destacaram por apresentarem a prática da acupuntura visando mostrar quais são seus efeitos colaterais e normas de biossegurança, os quais estão apresentados no Quadro 1, sistematizados de acordo com ano de publicação, autor, periódico e título. 
Pimenta FR, Leão LSNO, Pimenta FC. Controle de infecção: um requisito essencial na prática da acupuntura - revisão de $\begin{array}{llllll}\text { literatura. } & \text { Rev. } & \text { Eletr. } & \text { Enf. } & \text { [Internet]. }\end{array}$ http://www.fen.ufg. br/revista/v10/n3/v10n3a22.htm.

Quadro 1: Relação de 11 artigos relacionados a prática da acupuntura, visando mostrar quais são seus efeitos colaterais, segundo o ano de publicação, autores, periódico e título.

\begin{tabular}{|c|c|c|c|}
\hline Ano & Periódico & Autores & Título \\
\hline 2007 & Tanuseputro, Yip e Nolan & UTMJ & $\begin{array}{l}\text { Regulation of acupuncture and tradicional chinese } \\
\text { medicine in Ontorio }\end{array}$ \\
\hline 2004 & White & Acupunct Med & $\begin{array}{l}\text { A cumulative review of the range and incidence of } \\
\text { significant adverse events associated with } \\
\text { acupuncture }\end{array}$ \\
\hline 2003 & Ernst e Sherman & $\begin{array}{l}\text { J Gastroenterol } \\
\text { Hepatol }\end{array}$ & $\begin{array}{l}\text { Is acupuncture a risk factor for hepatitis? } \\
\text { Systematic review of epidemiological studies }\end{array}$ \\
\hline 2003 & Ernst, Strzyz e Hagmeister & $\begin{array}{l}\text { Complement Ther } \\
\text { Med }\end{array}$ & $\begin{array}{l}\text { Incidence of adverse effects during acupuncture } \\
\text { therapy - a multicentre survey }\end{array}$ \\
\hline 2003 & Lao, Hamilton, Fu e Berman & $\begin{array}{l}\text { Altern Ther Health } \\
\text { Med }\end{array}$ & $\begin{array}{l}\text { Is acupuncture safe? A systematic review of case } \\
\text { reports }\end{array}$ \\
\hline 2002 & Kaptchuk & Ann Intern Med & Acupuncture: theory, efficacy and practice \\
\hline 2001 & Walsh & Acupunct Med & Control of infection in acupuncture \\
\hline 2001 & Vincent & BMJ & The safety of acupuncture \\
\hline 2001 & $\begin{array}{l}\text { Yamashita, Tsukayama, } \\
\text { White, Tanno, Sugishita e } \\
\text { Ernst }\end{array}$ & $\begin{array}{l}\text { Complement Ther } \\
\text { Med }\end{array}$ & $\begin{array}{l}\text { Systematic review of adverse events following } \\
\text { acupuncture: the J apanese literature }\end{array}$ \\
\hline 1999 & Walsh, Maguire e Carrington & $\begin{array}{l}\text { Commun Dis } \\
\text { Public Health }\end{array}$ & Outbreak of hepatitis B in acupuncture clinic \\
\hline 1984 & Schmid, Hortling e Kammuller & Fortschr Med & $\begin{array}{l}\text { Inoculation hepatitis caused by acupuncture. } \\
\text { Clinical cases studied over } 9 \text {-year period }\end{array}$ \\
\hline
\end{tabular}

\section{RESULTADOS E DISCUSSÃO}

A análise do conteúdo dos 20 textos selecionados, será apresentada em dois tópicos: efeitos adversos na acupuntura e biossegurança e controle de infecção na acupuntura.

\section{Efeitos adversos na Acupuntura}

De acordo com os textos analisados, os efeitos adversos da acupuntura, são mínimos, destacando a segurança desta prática ${ }^{(2,4,9-10)}$. Verificou-se um maior número de publicações associadas a esse tópico após constatar uma maior aceitação da acupuntura por profissionais da saúde e pelos clientes em geral.

Os pricipais efeitos adversos devido a pratica da acupuntura, segundo a um estudo realizadocom dados publicados em língua inglesa, no período de 1965-1999, incluíam quadros de infecção, prevalecendo principalmente a hepatite, que pode ser evitado por treinamento rigoroso do profissional e limpeza adequada de agulhas, reduzindo o risco associado com esse procedimento ${ }^{(10)}$.

Tres estudos mostraram que os principais efeitos adversos que podem acontecer devido um procedimento de acupuntura são: tonturas e vertigens, hematoma local, pneumotórax, infecções locais, dermatite de contato, náuseas e vômitos, dor no local da punção com a agulha, mal-estar, traumas em órgãos, lesões em nervos, entre outros. Nas mãos de profissionais competentes, a probabilidade de complicações é pequena, sendo que a maioria delas podem ser evitadas ${ }^{(4,9-10)}$.

Dois estudos sistemáticos analisaram a taxa e a natureza dos efeitos adversos da acupuntura, ambos sugerem que a taxa de complicações é baixa e a maior parte das complicações é transitória como náuseas e vômitos, mal-estar, dor no local aplicação da agulha, tonturas, vertigens e dermatite de contato. A análise avaliou mais de 66000 tratamentos feitos por médicos, fisioterapeutas e acupunturistas tradicionais, sem nenhuma diferença significativa entre os grupos ${ }^{(11)}$.

As agulhas, principal ferramenta da acupuntura, são consideradas artigos críticos por penetrar a pele e tecidos do paciente. Devese, portanto, considerar o risco potencial deste artigo para carrear microrganismos causadores de infecção associado a sua utilização ${ }^{(12)}$. Cuidados especiais devem ser tomados na escolha da melhor agulha a ser utilizada, certificando-se da sua procedência, esterilização, embalagem e conservação antes do seu manuseio, evitando contaminações.

A associação da aquisição do vírus de hepatite por meio de procedimentos de acupuntura ainda é bastante questionável. Um estudo realizado em um hospital da Alemanha, 
Pimenta FR, Leão LSNO, Pimenta FC. Controle de infecção: um requisito essencial na prática da acupuntura - revisão de

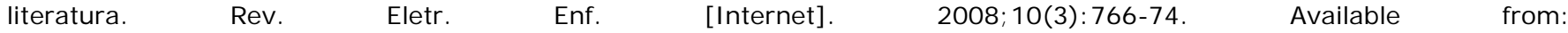
http://www.fen.ufg. br/revista/v10/n3/v10n3a22.htm.

entre 1976 e 1983, identificou 17 casos de hepatite (16 casos de hepatite $B$ e um caso de hepatite não A ou B - NANB) possivelmente associados à acupuntura. Verificaram que a desinfecção e esterilização incorreta, principalmente de agulhas utilizadas na sessão de acupuntura poderiam estar relacionadas à transmissão do vírus ${ }^{(13)}$.

Estudo que realizou uma revisão de relatos sobre a associação da acupuntura com a hepatite concluiu que existe uma associação entre o hepatite $C$ e acupuntura em alguns países, principalmente na Ásia, e enfatizaram a importância do uso de agulhas descartáveis ${ }^{(14)}$.

Outro possível fator de transmissão dos agentes infecciosos, como os vírus causadores de hepatites e de outras doenças infectocontagiosas, é o contato direto do próprio acupunturista com os seus pacientes. Um estudo realizado em uma clínica em Londres, por um período de três anos e meio, descreveu cinco casos de pacientes que contraíram o vírus da hepatite B (HBV). Desses casos, 1,7\% foram de pacientes tratados por um acupunturista portador do antígeno para o vírus da hepatite B (HBAg). Verificaram similaridade entre o vírus detectado no acupunturista com a de dois dos cinco pacientes. Outros nove pacientes atendidos pelo mesmo acupunturista apresentaram anticorpo para antígeno da hepatite $B$, mas possuíam outros fatores de risco para infecção pelo HBV. Não foi comprovada a via de transmissão, mas a contaminação por agulhas nos dois casos não pode ser descartada. Vale ressaltar que, nos casos de exposição ao HBV, foram utilizadas agulhas descartáveis. Portanto, recomenda-se a imunização dos acupunturistas contra possíveis doenças infecciosas, em especial ao $\mathrm{HBV}^{(15)}$.

De acordo com 12 estudos prospectivos ${ }^{(4)}$ que contou com a análise de mais de um milhão de tratamentos, os riscos de um efeito adverso sério associado com acupuntura foi estimado em $0,05 \times 10000$ tratamentos e de 0,55 $\times$ 10000 pacientes individuais, o que torna o risco muito baixo comparado com outros tratamentos médicos.

\section{Biossegurança e controle de infecção na Acupuntura}

Somente com a emergência de várias doenças infectocontagiosas e sua rápida disseminação pelo mundo, como por exemplo a epidemia da AIDS (Síndrome da Imunodeficiência Adquirida) na década de 80, vinculada a severidade e letalidade da doença, houve maior conscientização dos profissionais em relação ao controle de infecção e prevenção dessas doenças. Isso possibilitou ampliar seus conhecimentos quanto ao entendimento dos riscos reais a que estão expostos durante os procedimentos laborais e a importância de medidas protetoras ${ }^{(16)}$.

Dessa forma, por tratar-se de um material que penetra na pele e em vários outros tecidos, segundo a Resolução RDC (Resolução da Diretoria Colegiada) $\mathrm{n}^{\circ}$. 306, de 07 de dezembro de 2004, as agulhas de acupuntura pertencem ao grupo E - perfurocortantes, que são os objetos e instrumentos contendo cantos, bordas, pontas ou protuberâncias rígidas e agudas, capazes de cortar ou perfurar. Enquadram-se neste grupo: lâminas de barbear, bisturis, agulhas, escalpes, ampolas de vidro, lâminas e outros similares provenientes de serviços de saúde. A classificação adotada é baseada na Resolução CONAMA (Conselho Nacional do Meio Ambiente) no. . 5, de agosto de 1993; Resolução CONAMA no. 283, de julho de 2001; na NBR (Normas Brasileiras) no. 10004 da ABNT (Associação Brasileira de Normas Técnicas) - Resíduos Sólidos - Classificação, de setembro de 1987 e na NBR no. 12808 da ABNT, de janeiro de 1993, bem como em outros estudos e documentos pertinentes à matéria.

De acordo com a classificação proposta por estudo realizado em $1968^{(17)}$ a opção para a escolha do tipo de processamento de um artigo dependerá primariamente do risco potencial de aquisição de microrganismos causadores de infecção, associado a sua utilização. Desta forma, procedimentos invasivos em pele e mucosas adjacentes, nos tecidos subepiteliais, no sistema vascular e todos os que estejam diretamente conectados com este sistema, exigem artigos esterilizados - artigos críticos; o manuseio de mucosas íntegras ou pele não-íntegra exige esterilização, 
Pimenta FR, Leão LSNO, Pimenta FC. Controle de infecção: um requisito essencial na prática da acupuntura - revisão de

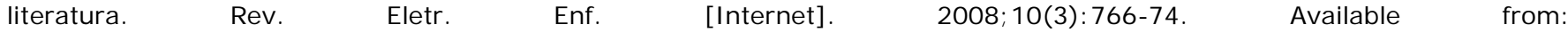
http://www.fen.ufg. br/revista/v10/n3/v10n3a22.htm.

desinfecção de alto nível ou intermediário artigos semicríticos; e o manuseio em pele íntegra ou de artigos que não entram em contato com o cliente requer no mínimo, limpeza ou desinfecção de baixo nível - artigos não $\operatorname{críticos}^{(18)}$.

Esse conjunto de condutas, denominado de biossegurança, tem sido intensamente estudado, avaliado, modificado e aperfeiçoado em virtude de ser um dos principais instrumentos para o controle da transmissão e disseminação dos microrganismos causadores de infecções, evitando a infecção cruzada ${ }^{(19)}$.

Estudo realizado (8) sinaliza que há necessidade de se criar uma regulamentação geral que requer registro sistemático dos profissionais de acupuntura, para facilitar e controlar sua fiscalização. Sem uma regulamentação previamente estabelecida, os inspetores públicos de saúde ficam limitados ao examinar as clínicas (exemplo: verificar somente registro e licença de funcionamento), enquanto outros profissionais que praticam acupuntura e que estão ligados aos seus conselhos, estão sujeitos aos padrões estabelecidos por sua profissão.

$\mathrm{Na}$ província de Ontário, até 2006, a profissão de acupunturista ainda não estava regulamentada, por isso preocupado com essa situação e para facilitar sua fiscalização, foi criado o Bill 50, denominado o "Tradicional Chinese Medicine Act, 2006" para regulamentar a prática da MTC e da acupuntura, enfocando a prática segura e efetiva e garantindo um nível de competência dos profissionais. Sua preocupação baseou-se principalmente na acupuntura, por envolver o uso de agulhas que penetram a pele, onde as práticas de controle de infecção são necessárias para prevenir a transmissão de microrganismos. Essa regulamentação objetivou criar uma unidade de saúde pública para realizar inspeção e reforçar os padrões de controle de infecção para reduzir os riscos de transmissão de doenças como HIV e $\mathrm{HBV}^{(8)}$.

Então, para boas práticas de atendimento a pacientes, devem-se conhecer as normas e os procedimentos de segurança, para minimizar os riscos de acidentes. Cabe ressaltar alguns cuidados durante a prática de acupuntura(1):
- Na introdução das agulhas, nunca tocar na lâmina das mesmas, o que pode ser evitado com a utilização do tubo guia (mandril). No caso das agulhas longas chinesas, deve-se utilizar luvas e gaze esterilizada;

- Na retirada das agulhas, ter atenção para evitar acidentes. As agulhas deverão ser descartadas em recipiente adequado conforme orientações sobre resíduos perfurocortantes.

As grandes causas de acidentes na prática da acupuntura estão relacionadas à instrução inadequada, supervisão ineficiente, mau uso dos Equipamentos de Proteção Individual (EPI), não observação de normas existentes, práticas inadequadas, planejamento falho e jornada excessiva de trabalho(1)

Apesar das agulhas serem consideradas como artigos críticos, existe poucos relatos de casos de contaminação devido ao uso de agulhas de acupuntura, mostrando que essa técnica é eficaz e segura, mas sempre se recomenda o uso de materiais descartáveis, devidamente esterilizados e embalados ${ }^{(2-4,14,20)}$.

Efeitos adversos associados ao tratamento com acupuntura são pouco prováveis. Os possíveis riscos relatados estão relacionados a traumas devidos inadequada aplicação da técnica pelo acupunturista (falta de capacitação do profissional) e em alguns episódios de infecção. Esses problemas poderiam ser minimizados por meio de higienização adequada das mãos, uso de luvas, anti-sepsia da pele e principalmente pelo uso de materiais descartáveis de boa procedência ${ }^{(4,8,20)}$.

A acupuntura é um procedimento seguro, desde que seja executada por profissionais hábeis e competentes. Estudos realizados no UK mostram que o risco com problemas é extremamente baixo, sendo que no geral, nenhuma infecção relacionada à complicações pela acupuntura tem sido relatadas recentemente. Para isso, procedimentos de controle de infecção devem continuar sendo desenvolvidos e aperfeiçoadas constantemente (20).

Casos de infecção bacteriana, como por exemplo septicemia e endocardite causada por Staphylococcus foram associados com o 
Pimenta FR, Leão LSNO, Pimenta FC. Controle de infecção: um requisito essencial na prática da acupuntura - revisão de

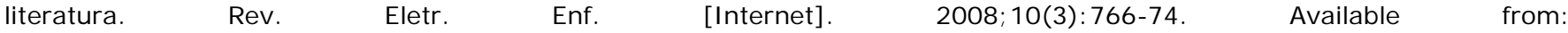
http://www.fen.ufg. br/revista/v10/n3/v10n3a22.htm.

tratamento de acupuntura, mas são raros. Ou seja, essa disseminação de uma pessoa para outra pode ocorrer, mas somente quando os processos de controle de infecção são falhos. Em particular, o uso de agulhas descartáveis excluem o risco de transmissão de microrganismos não apenas para os pacientes, mas também para o acupunturista que pode se contaminar ao se acidentar com as agulhas ao manuseá-las ${ }^{(20)}$.

\section{CONCLUSÃO}

Os textos analisados mostraram que a acupuntura é uma terapia segura e eficaz, desde que todas as normas de biossegurança sejam seguidas para evitar possíveis riscos na sua prática, principalmente a transmissão de microrganismos. Entretanto, é necessário que haja um controle de infecção rígido, com o cumprimento e atualização das normas, com a fiscalização sistemática pelos orgãos responsáveis.

Os principais efeitos adversos que ocorrem durante um procedimento de acupuntura destacados no material pesquisado, como por exemplo pneumotorax, dor no local da agulha, mal-estar, perfuração de estruturas anatômicas, lesões em nervos, infecção, entre outros, podem ser evitados desde que os acupunturistas sejam devidamente capacitados. A maior parte dos efeitos adversos encontrados na prática da acupuntura foram por falta de uso de material devidamente esterilizado e por despreparo dos profissionais.

Então, por ser uma técnica invasiva, que emprega agulhas como uma de suas principais ferramentas de aplicação, recomenda-se sempre o uso de agulhas descartáveis de boa procedência para evitar infecções cruzadas e rigidez na aplicação das normas de biossegurança.

\section{REFERÊNCI AS}

1. Nogueira IA, Maki, R. Manual de Biossegurança em Acupuntura. Rio de Janeiro: Secretaria de Estado de Saúde do Rio de Janeiro; 2003.

2. Yamashita H, Tsukayama H, White AR, Tanno $Y$, Sugishita C, Ernst E. Systematic review of adverse events following acupuncture: the
Japanese literature. Complementary Therapies in Medicine. 2001; 9(2): 98-104.

3. Kaptchuk TJ. Acupuncture: theory, efficacy, and practice. Ann Intern Med. 2002; 136(5): 374-83.

4. White A. A cumulative review of the range and incidence of significant adverse events associated with acupuncture. Acupuncture in medicine : journal of the British Medical Acupuncture Society. 2004;22(3): 122-133.

5. Ministério da Saúde. Política Nacional de Medicina Natural e Práticas Complementares PMNPC - junho de 2005. Brasília (Brasil): Ministério da Saúde; 2005.

6. Ministério da Saúde. Portaria № 971 de 3 de maio de 2006 - Edição Número 84 de 04/05/2006. Brasília (Brasil): Ministério da Saúde; 2006.

7 Yamamura Y. Acupuntura tradicional: a arte de inserir. $2^{a}$ edição. São Paulo: Roca; 2001.

8. Tanuseputro P, Yip G, Nolan L. Regulation of acupuncture and tradicional chinese medicine in Ontorio. The University of Toronto Medical Journal. 2007; 84(3):175-6.

9. Ernst $G$, Strzyz $H$, Hagmeister $H$. Incidence of adverse effects during acupuncture therapy-a multicentre survey. omplementary Therapies in Medicine 2003; 11(2): 93-7.

10. Lao L, Hamilton GR, Fu J, Berman BM. Is acupuncture safe? A systematic review of case reports. Alternative therapies in health and medicine. 2003; 9(1):72-83.

11. Vincent C. The safety of acupuncture. BMJ . 2001; 323: 467-8.

12. Center for Disease Control and Prevention $(C D C)$. Guidelines for Infection Control in Dental Health Care Setting. MMWR [Internet]. 2003 [cited 2005 oct 10];52(RR17):1-61. Available from:

http://www.cdc.gov/mmwr/preview/mmwrhtml/ rr5217a1.htm

13. Schmid E, Hortling G, Kammuller $H$. Inoculation hepatitis caused by acupuncture. Clinical cases studied over a 9-year period. Fortschritte der Medizin. 1984; 102(35):862-5.

14. Ernst E, Sherman KJ. Is acupuncture a risk factor for hepatitis? Systematic review of epidemiological studies. Journal of gastroenterology and hepatology. 2003; 18(11): 1231-6. 
Pimenta FR, Leão LSNO, Pimenta FC. Controle de infecção: um requisito essencial na prática da acupuntura - revisão de $\begin{array}{llllll}\text { literatura. Rev. } \quad \text { Eletr. } \quad \text { Enf. 2008; 10(3):766-74. Available from: } & \end{array}$ http://www. fen.ufg. br/revista/v10/n3/v10n3a22.htm.

15. Walsh B, Maguire H, Carrington D. Outbreak of hepatitis $B$ in acupuncture clinic. Communicable Disease and Public Health. $1999 ; 2(2): 79-81$.

16. Rodrigues MP, Domingos Sobrinho M, Silva EM. Os cirurgiões-dentistas e as representações sociais da Aids. Ciência e Saúde Coletiva. 2005; 10(2): 463-72.

17. Spaulding EH. Clinical disinfection of medical cirurgical material. In: Blawrence CA, Block SS. Disinfection, Sterilization and Preservation. Philadelphia: Lea \& Sebiger; 1968. p. 517-32

18. Kawagoe JY, Queiroz ML. Normas Básicas para a Realização de Procedimentos. In: Rodrigues EAA. Infecções Hospitalares: prevenção e controle. São Paulo: Sarvier; 1997. p. $58-66$

19. Pimenta FC, Ito IY, Lima SNM. Biossegurança em Endodontia. In: Estrela C, Figueiredo JAP. Endodontia: princípios biológicos e mecânicos. São Paulo: Artes Médicas; 1998. p. 386-438

20. Walsh B. Control of infection in acupuncture. Acupuncture in medicine : journal of the British Medical Acupuncture Society. 2001; 19(2): 109-11.

Artigo recebido em 21.12.07

Aprovado para publicação em 30.09.08 